\title{
DC Microgrid Voltage Stability by Dynamic Feedback Linearization
}

\author{
Filipe Perez ${ }^{1,4}$, Alessio Iovine ${ }^{2}$, Member, IEEE, Gilney Damm ${ }^{3}$, Member, IEEE, Paulo Ribeiro ${ }^{4}$,Fellow, IEEE \\ L2S Laboratory, CentraleSupélec, Paris-Saclay University, France, filipe.perez@12s.centralesupelec.fr ${ }^{1}$ \\ Efficacity R\&D Center, Paris, France, a.iovine@efficacity.com ${ }^{2}$ \\ IBISC Laboratory, Paris-Saclay University, France, gilney.damm@ibisc.fr ${ }^{3}$ \\ ISEE, Federal University of Itajubá, Itajubá, Brazil, pfribeiro@ieee.org ${ }^{4}$
}

\begin{abstract}
Recent developments in power electronics and renewable energy sources have brought the risen of Direct Current (DC) Microgrids. By power converters' utilization, a DC Microgrid is able to integrate renewables, storage systems, and other devices in a simpler topology, since the major part of the modern grid's compounds have DC nature. This paper proposes a control strategy aiming to regulate the voltage in the DC bus of a Microgrid by a supercapacitor. The control law is applied to a bidirectional boost converter that connects the supercapacitor to the DC grid. The control scheme is based on dynamical feedback linearization and consists in splitting the system in different time scale dynamics, creating consequently a simplified model. Simulations on Matlab/Simulink illustrate how the control strategy is able to regulate the DC voltage with good performance.
\end{abstract}

Index Terms-DC Microgrids control, voltage stability, Feedback linearization, Nonlinear control, Power converters.

\section{INTRODUCTION}

The impact of distributed generation in electrical grids has been a widespread concern. This impact is primary related to the intermittent nature of renewable energy sources (renewables) and the harmonic distortions caused by their converters. Many solutions have been proposed to improve the integration of new technologies in the power grid [1], [2]. Direct Current (DC) Microgrids integrating renewables and storages are the solution that is becoming increasingly interesting, as they allow autonomy with respect to the main grid. Also, they bring a better integration of their parts, since most electronic devices (PV, batteries, electric vehicles ...) have a DC nature [3], [4].

Nowadays, several examples of DC Microgrids exist in marine, aviation, automotive, and manufacturing industries. For all of them, it is extremely important that a Microgrid presents reliability and proper control operation [5], [6]. In this context, nonlinear theory allows for a more realistic grid modeling and a more effective stability analysis [7], [8], [9], [10]. The utilization of nonlinear control techniques may also improve power flow performances in the Microgrid, since the system is not restricted by an operating point and there is the possibility to work in a wider region of operation, considering just the physical limitations of the system as restrictions. A drawback of the use of nonlinear control technique is the increased complexity of the resulting control law, which is sometimes difficult to be implemented [7].

In this work, a control scheme for a DC Microgrid is introduced, to the purpose to control power converters when problems related to non-minimum phase system characteristics appear because of control targets and converters' topology. Such problems limit state feedback utilization in some electrical schemes: for example, a boost configuration for a supercapacitor in charge of controlling the voltage in the DC bus does not allow the utilization of the classical feedback linearization. On the contrary, the here proposed dynamical feedback linearization technique is based on a system's equilibrium value that is calculated in advance and allows state feedback utilization.

To the target to reduce size and complexity, a simplified model for the DC grid configuration will be used, based on a Thévenin equivalent circuit. A dynamical feedback linearization control based on the simplified model and on singular perturbation analysis is developed; only the voltage equilibrium point will be required, allowing for a simpler control law. This approach avoids the problems related to the non-minimum phase characteristics when the target is the output voltage control in a boost converter [8], [11].

This paper is organized as follows: Section II introduces the considered simplified model, while Section III describes the adopted control law. Simulations are outlined in Section IV and conclusions are provided in Section V.

\section{MICROGRID MODELING}

In [7] a DC Microgrid composed by a variable load, a PV array for power generation and two energy storage systems (a battery and a supercapacitor) operating at different time scales for power management has been analyzed and a proper distributed nonlinear control has been proposed as solution. With respect to [7], in this paper a new control scheme for regulating the DC voltage bus in the Microgrid is introduced, taking into account a more interesting electrical scheme. Indeed, the supercapacitor with a buck configuration may be unfavorable, since its voltage needs to be higher than the DC grid voltage and consequently it has a more expensive construction [12]. A DC/DC bidirectional-boost is here considered to connect the supercapacitor and the DC grid performing the same DC grid voltage stability operations as in [7], i.e. the supercapacitor is in charge of keeping the DC voltage at the desired level while the battery is used as energy reservoir. Furthermore, the variable loads is here connected to the DC grid by a DC/DC converter. 


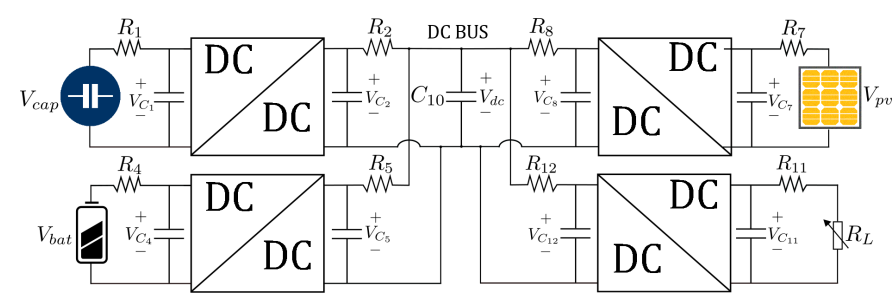

Fig. 1. The DC Microgrid integrating the PV array, the load and the two energy storage systems operating at different time scales.

Fig. 1 introduces the considered DC Microgrid, which is composed by a supercapacitor, a battery, a PV array and a variable load. Each device is connected to a DC bus by a DC/DC converter: they are bidirectional-boost ones for supercapacitor and battery, a boost for PV and a buck for the load. Hypothesis on the good and proper size of the all system's components are done as in [7].

The contribution of this paper is to provide a simpler control scheme for the DC bus of a Microgrid with respect to the one introduced in [7], which has been shown to deal in a more effective way with the system interconnection with respect to classical PI control. Part of the contribution relies also in the capability to use a boost converter for stabilizing purposes. Indeed, by controlling the output voltage in the boost converter, it is possible to bring the grid voltage $V_{d c}$ to a desired value.

\section{A. Thévenin Equivalent Circuit}

Assumption 1: In the Microgrid, we consider that the voltage of the output capacitor of the PV array, the battery and the load are considered as piecewise constant plus a slowly timevarying disturbance.

This because the battery is controlled in a time-scale of seconds according to power flow regulation, while the load is in general a sum of switching elements in addition to slowly (seconds) varying ones. Finally, in the PV case, the disturbances come from the solar radiance, where the solar variations is much slower than the voltage control time-scale. The time varying disturbances will be neglected; the effect of this will be an additive disturbance in a linear stable closed loop system, which will be compensated by the robustness of the controller. Nevertheless, there will be an arbitrarily small slowly varying disturbance that will not bring the system outside of the operation margin defined around the desired equilibrium point.

Therefore, we can apply Thévenin theorem on those devices to obtain an equivalent circuit. The DC bus is the coupling point for the devices integrated in the system, and it is here modeled as a capacitance. The Thévenin equivalent circuit is used to substitute PV, battery and load by a voltage source $V_{t h}$ and a resistance $R_{t h}$.

Thévenin voltage $V_{t h}$ is calculated according to PV, battery and load system in open circuit.

$$
V_{t h}=R_{t h}\left[\frac{V_{C_{5}}}{R_{5}}+\frac{V_{C_{8}}}{R_{8}}+\frac{V_{C_{12}}}{R_{12}}\right]
$$

where $V_{C_{5}}, V_{C_{8}}$ and $V_{C_{12}}$ are converters' output voltage on PV, battery and load, respectively presented in Fig. 1. They

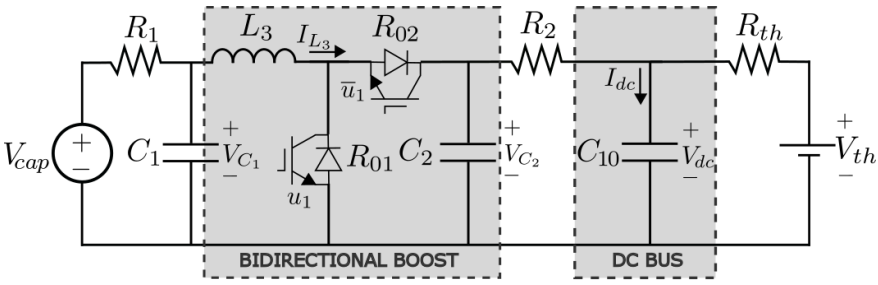

Fig. 2. The simplified electrical model of the DC Microgrid.

are considered according to assumption 1; consequently, the resulting voltage $V_{t h}$ can be considered piecewise constant plus slowly time-varying as well.

The equivalent Thévenin resistance $R_{t h}$ is calculated as

$$
R_{t h}=\frac{R_{5} R_{8} R_{12}}{R_{5} R_{8}+R_{5} R_{12}+R_{8} R_{12}}
$$

where $R_{5}, R_{8}$ and $R_{12}$ are the cable losses of the converters of PV, battery and load respectively.

\section{B. Simplified Microgrid Model}

Fig. 2 depicts the Microgrid's simplified electrical model due to considerations in Section II-A. It is composed by the converter that is connected to the supercapacitor, a capacitor representing the DC bus and the Thévenin equivalent circuit. $V_{c a p}$ is the voltage on the supercapacitor, $V_{d c}$ is the voltage on the DC bus and $C_{10}$ is the capacitance of DC bus. $R_{1}$ and $R_{2}$ are the resistances representing cable losses, while $R_{01}$ and $R_{02}$ are switch losses of the semiconductors, where $R_{01}=R_{02}$. $V_{C_{1}}$ is the voltage of capacitor $C_{1}, V_{C_{2}}$ is the voltage of capacitor $C_{2}$ and $I_{L_{3}}$ is the current of inductor $L_{3}$ : they are the state variables of the system. The control input $u_{1}$ is the converter duty cycle, where $u_{1} \in[0,1]$.

The equivalent circuit is expressed using state space average model as follows:

$$
\left\{\begin{array}{l}
\dot{V}_{C_{1}}=\frac{1}{R_{1} C_{1}} V_{c a p}-\frac{1}{R_{1} C_{1}} V_{C_{1}}-\frac{1}{C_{1}} I_{L_{3}} \\
\dot{V}_{C_{2}}=\frac{1}{R_{2} C_{2}} V_{d c}-\frac{1}{R_{2} C_{2}} V_{C_{2}}+\frac{1}{C_{2}} I_{L_{3}}\left(1-u_{1}\right) \\
\dot{I}_{L_{3}}=\frac{1}{L_{3}} V_{C_{1}}-\frac{1}{L_{3}} V_{C_{2}}\left(1-u_{1}\right)-\frac{R_{01}}{L_{3}} I_{L_{3}} \\
\dot{V}_{d c}=\frac{1}{C_{10} R_{2}}\left(V_{C_{2}}-V_{d c}\right)+\frac{1}{C_{10} R_{t h}}\left(V_{t h}-V_{d c}\right)
\end{array}\right.
$$

\section{MICROGRID CONTROL}

According to its multi-time scale characteristics, the system introduced in (3) can be split in two subsystems; one subsystem is composed only by the current $I_{L_{3}}$, while the other one represents the three voltages $V_{C_{1}}, V_{C_{2}}$ and $V_{d c}$. To correctly address the desired actions, three steps need to be envisaged for performing the control algorithm:

1) To introduce a stabilizing controller for the subsystem representing the current $I_{L_{3}}$. Indeed, this subsystem is controllable and is allocated to have faster dynamics with respect to the other one. The first target will be to steer the controllable faster dynamics $I_{L_{3}}$ for bringing it to a desired trajectory $I_{L_{3}} \rightarrow I_{L_{3}}^{*}$, where $I_{L_{3}}^{*}$ is yet to be designed. To develop the controller for the fast 
dynamics separately from the slow dynamics some mild conditions are considered, used and verified (see [13]).

2) To define the operating region where the controller that has been introduced in the previous step is able to work with respect to the reference $I_{L_{3}}^{*}$, which clearly depends on the state of the system and of the desired value has to be imposed.

3) To calculate the reference $I_{L_{3}}^{*}$ for the current dynamics, according to the simplified model of the DC grid and on the desired equilibrium point for the capacitor $V_{C_{2}}^{*}$, i.e. $V_{C_{2}}^{*}$. This reference is obtained by considerations about singular perturbation analysis and the Thévenin equivalent circuit. Thus, $I_{L_{3}}^{*}$ will be deduced from the desired equilibrium point of the output capacitor $V_{C_{2}}^{*}$.

With this methodology, it is possible to avoid the problems related to the non-minimum phase characteristics of the system that is due to the structure of the boost converter. The target will be to steer $V_{C_{2}}$ to a desired $V_{C_{2}}^{*}$, providing DC bus voltage regulation.

\section{A. Feedback Linearization for $I_{L_{3}}$}

The first step is to control the current $I_{L_{3}}$ in the supercapacitor converter tracking its reference $I_{L_{3}}^{*}$, which is now supposed to be know, as well as its evolution over the time. From singular perturbation analysis [9], we can consider the states $V_{d c}, V_{C_{1}}$ and $V_{C_{2}}$, as constant in this part and then just study the subsystem describing the current. According to the control objective, the output is defined as $y_{1}=I_{L_{3}}$. The system is a single-input single-output one:

$$
\left\{\begin{array}{l}
\dot{x}_{1}=f_{1}\left(x_{1}\right)+g_{1} u_{1} \\
y_{1}=I_{L_{3}}
\end{array}\right.
$$

where $x_{1} \triangleq\left(\begin{array}{llll}V_{C_{1}} & V_{C_{2}} & I_{L_{3}} & V_{d c}\end{array}\right)^{T}, f_{1}$ and $g_{1}$ defined in (3), and where the Lie derivative of the output with respect to $g_{1}$ is calculated as:

$$
J=L_{g_{1}}\left(I_{L_{3}}\right)=-\frac{V_{C_{2}}}{L_{3}}
$$

Since $V_{C_{2}}$ is always positive by technological reasons, $J$ is non-singular and therefore a nonlinear feedback linearizing control for the control input $u_{1}$ can be written as:

$$
u_{1}=J^{-1}\left[v_{3}-\frac{1}{L_{3}}\left(V_{C_{1}}-V_{C_{2}}-R_{01} I_{L_{3}}\right)\right]
$$

where $v_{3}$ is the additional input to be designed. It must to be noticed that $V_{C_{1}}$ and $V_{C_{2}}$ are considered constant because of singular perturbation motivations.

The next step is to design the additional input $v_{3}$ to the purpose to bring the output $I_{L_{3}}$ to its desired trajectory $I_{L_{3}}^{*}$ :

$$
\begin{aligned}
v_{3} & =\dot{I}_{L_{3}}^{*}-K_{3}\left(I_{L_{3}}-I_{L_{3}}^{*}\right)-K_{3}^{\alpha} \alpha_{3} \\
\dot{\alpha_{3}} & =I_{L_{3}}-I_{L_{3}}^{*}
\end{aligned}
$$

where $K_{3}$ and $K_{3}^{\alpha}$ are positive constants, chosen in a way such that the multi-time scale nature of the dynamics of $I_{L_{3}}$ with respect to the voltages is preserved, or even amplified. By substituting (6), (7) and (8) in (4), the resulting dynamics is linear and stable, where $\alpha_{3}$ is the auxiliary variable that can be interpreted as the integral part in a PI controller. $\dot{I}_{L_{3}}^{*}$ is the time-derivative of the desired $I_{L_{3}}^{*}$ that has to be designed; then, both are known.

\section{B. Analysis of stability and operating regions}

The states $V_{C_{1}}, V_{C_{2}}$ and $V_{d c}$ presented in (3) are the zero dynamics of the whole system with respect to output $I_{L_{3}}$. Due to the multi-time scale characteristics with respect to the current $I_{L_{3}}$, the linear dynamics of $V_{C_{1}}$ is shown to be exponentially stable thanks to the fact that it is a linear system with a negative eigenvalue and two disturbances $\left(V_{P V}\right.$ and $I_{L_{3}}$ ) that will only modify its equilibrium point. For these reasons, in this section a stability study is presented for just the two variables representing the interconnected system, i.e. $V_{C_{2}}$ and $V_{d c}$.

The calculated equilibrium points for $V_{C_{2}}$ and $V_{d c}$ are:

$$
\begin{gathered}
V_{C_{2}}^{e}=\frac{V_{d c}}{2} \pm \frac{1}{2} \sqrt{V_{d c}^{2}+4 R_{2} I_{L_{3}}^{*}\left(V_{C_{1}}-R_{01} I_{L_{3}}^{*}\right)} \\
V_{d c}^{e}=\frac{R_{2} R_{t h}}{R_{2}+R_{t h}}\left[\frac{V_{C_{2}}}{R_{2}}+\frac{V_{t h}}{R_{t h}}\right]
\end{gathered}
$$

As stated in (10), the variable $V_{d c}$ has just one equilibrium point while $V_{C_{2}}$ has two equilibrium points (see (9)); the linearization technique will be used for stability analysis in these equilibrium points. The resulting Jacobian linearization matrix $A$ is:

$$
A=\left[\begin{array}{cc}
-\frac{1}{R_{2} C_{2}}-\frac{I_{L_{3}}^{*}\left(V_{C_{1}}-R_{01} I_{L_{3}}^{*}\right)}{C_{2} V_{C_{2}}^{e}{ }^{2}} & \frac{1}{R_{2} C_{2}} \\
\frac{1}{R_{2} C_{10}} & -\frac{1}{C_{10}}\left(\frac{1}{R_{2}}+\frac{1}{R_{t h}}\right)
\end{array}\right]
$$

and its eigenvalues can be written as:

$$
\lambda_{1,2}=-\frac{b}{2} \pm \frac{1}{2} \sqrt{b^{2}-4 c}
$$

where:

$$
\begin{aligned}
& b=\frac{1}{R_{2} C_{2}}+\frac{I_{L_{3}}^{*}}{C_{2} V_{C_{2}}^{e}}\left(V_{C_{1}}-R_{01} I_{L_{3}}^{*}\right)+\frac{1}{C_{10}} \frac{R_{2}+R_{t h}}{R_{2} R_{t h}} \\
& c=\frac{1}{C_{2} C_{10}}\left[\frac{R_{2}+R_{t h}}{R_{2} R_{t h}} \frac{I_{L_{3}}^{*}}{V_{C_{2}}^{e}}\left(V_{C_{1}}-R_{01} I_{L_{3}}^{*}\right)+\frac{1}{R_{2} R_{t h}}\right]
\end{aligned}
$$

A stability region can then be established with respect to the values of $b$ and $c$; in the following, a dedicated analysis is introduced according to their signs:

1) For $b>0$ and $c>0$ : the eigenvalues are such that $\operatorname{Re}\left[\lambda_{1,2}<0\right]$, and consequently the equilibrium points of the zero dynamics are stable. By considerations on the above inequalities, we can find out a region related to the value of $I_{L_{3}}^{*}$. The first region is given by $b>0$ :

$$
\frac{V_{C_{1}}}{2 R_{01}}-\frac{1}{2 R_{01}} \sqrt{\Delta_{1}}<I_{L_{3}}^{*}<\frac{V_{C_{1}}}{2 R_{01}}+\frac{1}{2 R_{01}} \sqrt{\Delta_{1}}
$$

where $\Delta_{1}=V_{C_{1}}{ }^{2}+4 R_{01} V_{C_{2}}^{e} \frac{C_{10} R_{t h}+C_{2}\left(R_{2} R_{t h}\right)}{R_{2} C_{2} R_{t h} C_{10}}$.

The second region is given considering $c>0$, and it can be expressed as:

$$
\frac{V_{C_{1}}}{2 R_{01}}-\frac{1}{2 R_{01}} \sqrt{\Delta_{2}}<I_{L_{3}}^{*}<\frac{V_{C_{1}}}{2 R_{01}}+\frac{1}{2 R_{01}} \sqrt{\Delta_{2}}
$$


with $\Delta_{2}=V_{C_{1}}{ }^{2}+4 R_{01} V_{C_{2}}^{e} \frac{1}{R_{2}+R_{t h}}$. Since (15) is in (14), the intersection of (14) and (15) is given by (15), which is the region of stability for $I_{L_{3}}^{*}$.

2) For $b \geq 0$ and $c \leq 0$ : at least one eigenvalue has a positive real part $\operatorname{Re}\left[\lambda_{1,2} \geq 0\right]$, then the system is not stable.

3) For $b \leq 0$ : there is at least one eigenvalue with positive real part $\operatorname{Re}\left[\lambda_{1,2} \geq 0\right]$ where the zero dynamics are not stable.

The introduced analysis describes the operating regions where the control of $I_{L_{3}} \rightarrow I_{L_{3}}^{*}$ is stable, both for the charge and the discharge of the supercapacitor, and implicitly provides some bounds for the reference $I_{L_{3}}^{*}$. In fact, it is necessary to realize that they reflect the physical limitations of the converter. So, the two zero dynamics are stable according to the aforementioned operating regions. In the following of this paper, authors make the reasonable assumption not to violate the bounds and then to operate in the region of stability for the systems interconnection.

\section{Reference calculation}

Here, the automatic procedure for obtaining the reference $I_{L_{3}}^{*}$ is described. A desired fixed value for the dynamics $V_{C_{2}}$ is supposed to be considered as reference, i.e. $V_{C_{2}}^{*}$. Starting from it, considerations about the dynamics of the system will be used to calculate a slowly varying desired reference $I_{L_{3}}^{*}$ whose derivative has a minimum effect in the previously designed current control. In this paper, the reference value for the dynamics $V_{d c}$ will be considered for $V_{C_{2}}^{*}$, i. e. $V_{d c}^{*}$. It is important to remark that the utilization of the fixed value $V_{d c}^{*}$ as desired reference for $V_{C_{2}}$ instead of the time-varying $V_{d c}$ will induce a steady state error in $V_{d c}$, which is the voltage drop in $R_{2}$ (cable loss). This because the capacitor's voltage will not be driven to the desired bus voltage in a direct way. Nevertheless, the trade off between this small error (proportional to the resistance of the bus connecting $V_{C_{2}}$ and $V_{d c}$ ) and a greatly simplified control design is very convenient.

Following the theory used in [9] and [14], and according to what stated in Section III-A, the controller designed in the previous step will assure that $I_{L_{3}}$ quickly converges to $I_{L_{3}}^{*}$. In this way, it is created a two time scales behaviour in the system, one for the $I_{L_{3}}$ current and another for the remaining states (mainly the voltages). For this reason, and following [9] and [14], the dynamics of $V_{C_{2}}$ presented in (3) can then be replaced by:

$$
\dot{V}_{C_{2}}=\frac{1}{R_{2} C_{2}} V_{d c}-\frac{1}{R_{2} C_{2}} V_{C_{2}}+\frac{1}{C_{2}} I_{L_{3}}^{*}\left(1-u_{1}^{*}\right)
$$

where $u_{1}^{*}=1-\frac{1}{V_{C_{2}}}\left[V_{C_{1}}-R_{01} I_{L_{3}}^{*}\right]$.

We desire to develop a reference $I_{L_{3}}^{*}$ such that $V_{C_{2}}$ is stabilized at $V_{C_{2}}^{*}$. To do that, the dynamics $V_{C_{2}}$ is considered at the equilibrium substituting $u_{1}^{*}$ in (16). This dynamics is then used to calculate the good reference for $I_{L_{3}}^{*}$. Furthermore, a virtual input $v_{d}$ has to be added to the system:

$$
\left\{\begin{array}{l}
\dot{V}_{C_{2}}=\frac{1}{R_{2} C_{2}}\left(V_{d c}-V_{C_{2}}\right)+\frac{I_{L_{3}}^{*}}{C_{2} V_{C_{2}}}\left[V_{C_{1}}-R_{01} I_{L_{3}}^{*}\right] \\
\dot{I}_{L_{3}}^{*}=v_{d}
\end{array}\right.
$$

Here $v_{d}$ is the virtual input, and it is needed to impose a desired dynamics to $I_{L_{3}}^{*}$. The system in (17) is in the following standard form

$$
\left\{\begin{array}{l}
\dot{x}_{0}=f\left(x_{0}, z\right)+g_{0} v_{d} \\
y_{2}=V_{C_{2}}
\end{array}\right.
$$

where $x_{0} \triangleq\left(\begin{array}{ll}V_{C_{2}} & I_{L_{3}}^{*}\end{array}\right)^{T}, z \triangleq\left(\begin{array}{ll}V_{C_{1}} & V_{d c}\end{array}\right)^{T}$ is continuous and know as well as its time derivative and being $v_{d}$ the control input. By defining $V_{C_{2}}$ as output, the relative degree is equal to 2 and consequently the considered system is fully controllable. The following Lie derivatives can be deduced as:

$$
\begin{gathered}
\dot{V}_{C_{2}}=L_{f_{0}}^{1}\left(V_{C_{2}}\right) \\
\ddot{V}_{C_{2}}=\frac{\dot{V}_{d c}}{R_{2} C_{2}}-\dot{V}_{C_{2}}\left[\frac{1}{R_{2} C_{2}}+\frac{V_{C_{1}} I_{L_{3}}^{*}-R_{01} I_{L_{3}}^{*}{ }^{2}}{C_{2} V_{C_{2}}{ }^{2}}\right]+ \\
+\frac{1}{C_{2} V_{C_{2}}} I_{L_{3}}^{*} \dot{V}_{C_{1}}+\frac{1}{C_{2} V_{C_{2}}}\left(V_{C_{1}}-2 R_{01} I_{L_{3}}^{*}\right) \dot{I}_{L_{3}}^{*} \\
\ddot{V}_{C_{2}} \triangleq L_{f_{0}}^{2}\left(V_{C_{2}}\right)+L_{g_{0}} L_{f_{0}}^{1}\left(V_{C_{2}}\right) v_{d}
\end{gathered}
$$

To introduce a desired synthetic input $\theta_{d}$, the input $v_{d}$ can be designed as:

$$
v_{d}=\frac{1}{L_{g_{0}} L_{f_{0}}^{1}\left(V_{C_{2}}\right)}\left[\theta_{d}-L_{f_{0}}^{2}\left(V_{C_{2}}\right)\right]
$$

where the additional input is chosen using linear techniques to give the desired dynamics for $V_{C_{2}}$.

$$
\theta_{d}=-K_{2}\left(\dot{V}_{C_{2}}-\dot{V}_{C_{2}}^{*}\right)+K_{2}^{\alpha}\left(V_{C_{2}}-V_{C_{2}}^{*}\right)
$$

As the reference $V_{C_{2}}^{*}$ is considered slowly time varying (and most often constant), its time derivative can be neglected. By substituting (22) and (23) in the system (17), the following closed-loop system is obtained:

$$
\left[\begin{array}{c}
\dot{V}_{C_{2}} \\
\ddot{V}_{C_{2}}
\end{array}\right]=\left[\begin{array}{cc}
0 & 1 \\
-K_{2}^{\alpha} & -K_{2}
\end{array}\right]\left[\begin{array}{c}
V_{C_{2}}-V_{C_{2}}^{*} \\
\dot{V}_{C_{2}}
\end{array}\right]
$$

Here the convergence speed of the voltage $V_{C_{2}}$ can be imposed by pole placement considerations. The gains $K_{2}$ and $K_{2}^{\alpha}$ need then to be properly chosen.

The steps presented above can now be stated in the form of the following theorem.

Theorem 3.1: System (3) is asymptotically stable at the desired equilibrium point under utilization of the control law (6) and of a reference trajectory for the current given by (22) in (17). The gains $K_{2}, K_{2}^{\alpha}, K_{3}$ and $K_{3}^{\alpha}$ in (24) and (7) have to be suitably chosen to define the proper control action.

The overall strategy may be seen as composed in two parts: at first, the compensation of the nonlinearities takes places to enlarge the operating region, and then the steering of the resulting system to its equilibrium point by a PI through pole placement procedure takes place, with the advantage to easily be able to chose the gains.

Remark 1: The term $V_{C_{1}}-2 R_{01} I_{L_{3}}^{*}$ is always positive since the voltage $V_{C_{1}}$ is always much larger than the voltage dissipation represented by $2 R_{01} I_{L_{3}}^{*}$, so the input $v_{d}$ is always nonzero. 
TABLE I

BOOST CONVERTER PARAMETERS

\begin{tabular}{c|c||c|c||c|c}
$R_{1}$ & $0.1 \Omega$ & $R_{2}$ & $0.1 \Omega$ & $L_{3}$ & $3.3 \mathrm{mH}$ \\
\hline$C_{1}$ & $100 \mathrm{mF}$ & $C_{2}$ & $10 \mathrm{mF}$ & $R_{01}$ & $10 \mathrm{~m} \Omega$
\end{tabular}

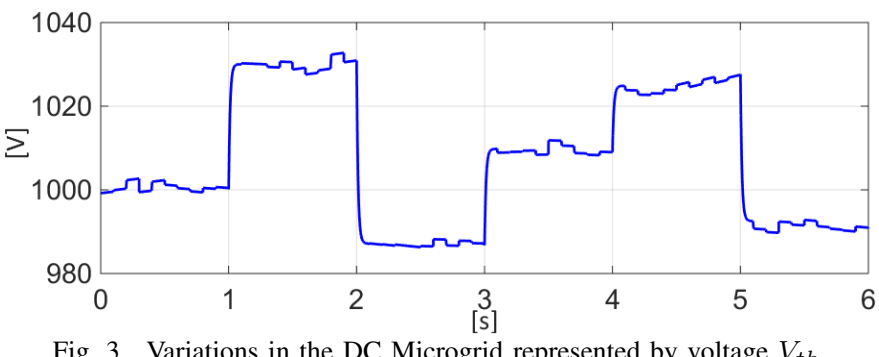

Remark 2: A reference trajectory $I_{L_{3}}^{*}$ is deduced from (17) model and its dynamics can be controlled by pole placement with the input $\theta_{d}$. From [9], one can see that the use of singular perturbation to obtain the simplified model is valid inside an operating region that is given by the time scale ratio from the two subsystems. As a consequence, the desired imposed dynamics should be chosen such as to obtain a suitable operation region for the DC Microgrid.

Remark 3: In the proposed control the time derivative of $V_{t h}$ is neglected. This because its components are either piecewise constants (battery and part of loads) or slower varying (solar and remaining loads) compared to the supercapacitor dynamics. The result of neglecting them is that there will be an additive time varying disturbance in the feedback linearized system. Such situation is standard and will impose errors in $V_{d c}$ and $V_{C_{2}}$ that will be arbitrarily attenuated by the pole placement.

\section{Simulation RESUlTS}

The electrical system in Fig. 2 has been simulated on Matlab/Simulink using the SimPowerSystem toolbox to test the proposed controller. The initial conditions are $I_{L_{3}}(0)=0 \mathrm{~A}$, $V_{C_{1}}(0)=400 \mathrm{~V}, V_{C_{2}}(0)=1000 \mathrm{~V}, V_{d c}(0)=1000 \mathrm{~V}$ and $V_{\text {cap }}(0)=400 \mathrm{~V}$. The capacitance of the supercapacitor is $50 F$ with a nominal voltage of $400 \mathrm{~V}$, in order to have the capability of being in charge of the voltage stabilization. Table I shows the used parameters. The switching frequency for PWM is $f_{s}=10 \mathrm{kHz}$.

A constant value of $1000 \mathrm{~V}$ is considered as reference for $V_{C_{2}}^{*}$; it is the desired value to be obtained for $V_{d c}$. This approach does not control $V_{d c}$ directly, but it provides DC grid voltage regulation by maintaining $V_{d c}$ in a granted region (with an error smaller than 5\%) with smooth oscillations.

The voltages of the PV array, battery and load that are represented by the Thévenin voltage are depicted in Fig. 3, where $V_{t h}$ is piecewise constant with realistic time-varying oscillations. The calculated value of Thévenin resistance is $R_{t h}=0.33 \Omega$. The supercapacitor voltage $V_{\text {cap }}$ is shown in Fig. 4. It reproduces the charge and discharge operations according to voltage variations. The supercapacitor absorbs the exceeding energy and supplies the missing one, balancing the power flow in the DC bus. Fig. 5 describes the voltage of the capacitor $C_{1}$, which varies according to $V_{c a p}$ and the required current $I_{L_{3}}^{*}$.

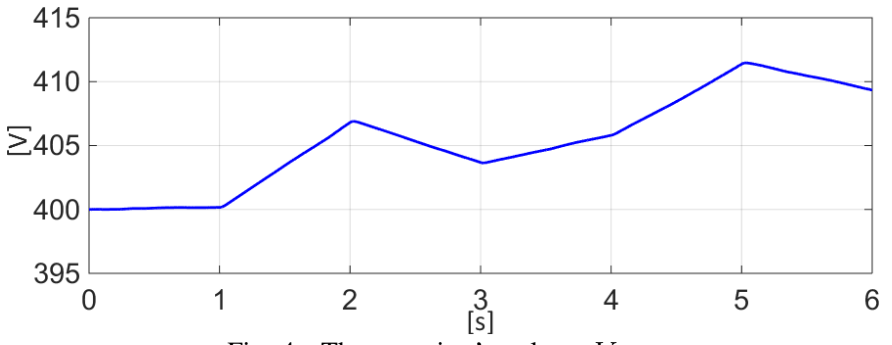

Fig. 4. The capacitor's voltage $V_{c a p}$.

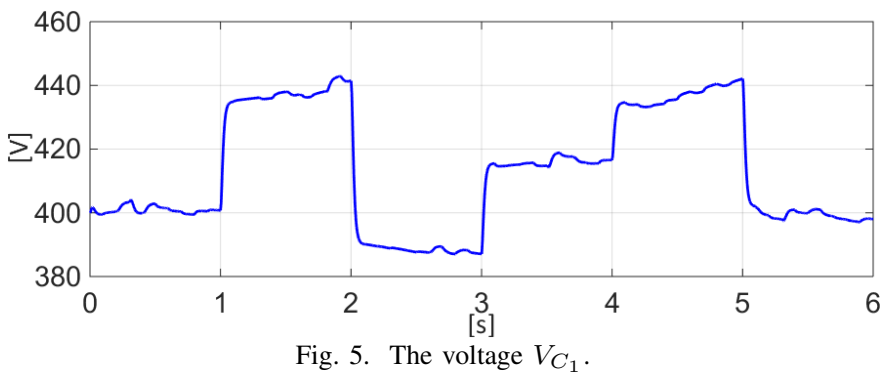

Fig. 6 introduces the dynamical behaviour of the voltage $V_{C_{2}}$ with respect to its reference $V_{C_{2}}^{*}$, which is constant. To the purpose to show the correctness of the hypothesis used in the paper, Fig. 6 also introduces a value $V_{C_{2} n}$ which represents $V_{C_{2}}$ when the current $I_{L_{3}}$ is in its reference value: since the two behaviours are similar, it is possible and reasonable to use $V_{C_{2} n}$ instead of $V_{C_{2}}$ for the calculation of $I_{L_{3}}^{*}$, as seen in Section III-C. By a comparison between Figures 3 and 6 , it is possible to note that $V_{C_{2}}$ has some small transients when $V_{t h}$ varies. The ripples are caused by the converter switching; nevertheless, they can be considered moderate. Also, the oscillations for $V_{C_{2}}$ are about $0.5 \%$. Fig. 7 depicts the behaviours of the current $I_{L_{3}}$ and its reference $I_{L_{3}}^{*}$. Obviously, the dynamics of the current varies according to the voltage level in $V_{t h}$ with respect to $V_{d c}$. The proper calculation of the reference $I_{L_{3}}^{*}$ is shown in Fig 8, where the DC grid voltage is depicted in p.u..Since the steady state error on $V_{d c}$ is less than $2 \%$, the reference $I_{L_{3}}^{*}$ has been properly obtained to perform the desired result, i.e. $V_{d c}$ results to be regulated inside the

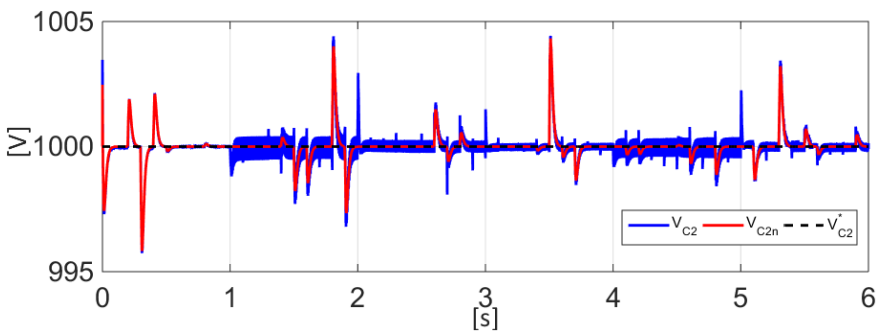

Fig. 6. The voltage $V_{C_{2}}$, its reference $V_{C_{2}}^{*}$ and the value $V_{C_{2} n}$.

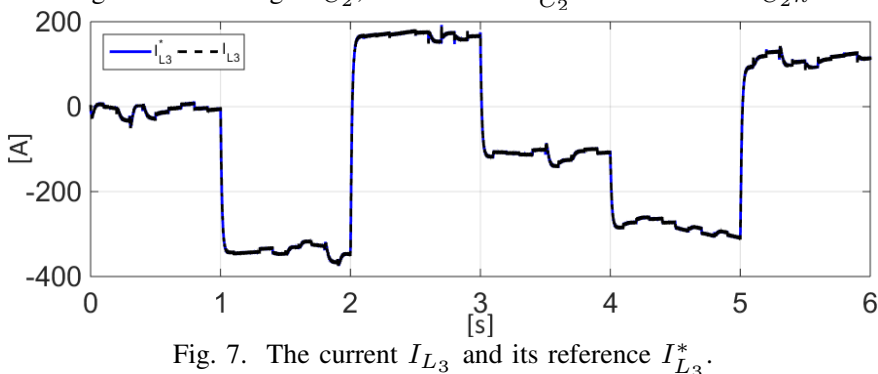




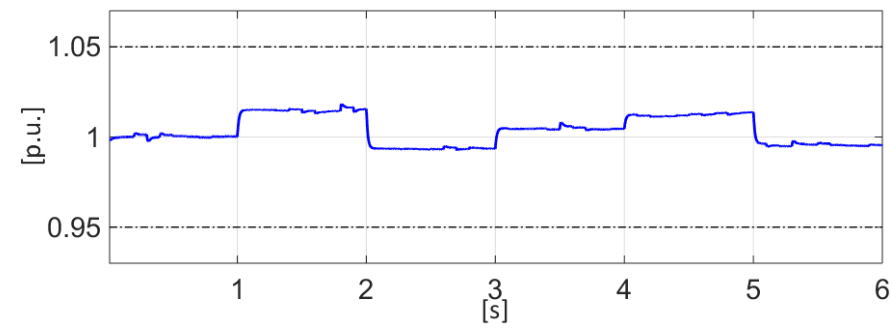

Fig. 8. The DC grid voltage $V_{d c}$ and its operation bounds in p.u. $\left(V_{d c}\right.$, base $\left.=1000 \mathrm{~V}\right)$, with a reference value of $V_{d c}^{*}=1000 \mathrm{~V}$..

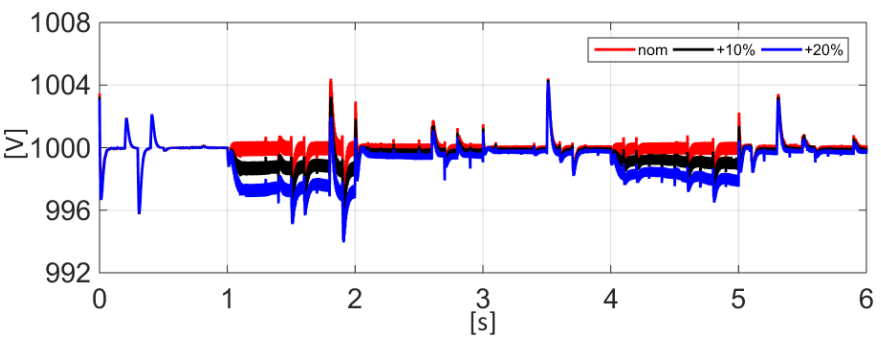

Fig. 9. The voltage $V_{C_{2}}$ with respect to the parameters errors.

grant region given by the grid requirements (+/- 5\%). The DC bus voltage has been stabilized inside the grant region.

\section{A. Robustness of the proposed control}

To the purpose to better validate the control performance, simulations illustrating the robustness of the proposed control action will now be introduced. An error percentage is considered in the knowledge of some system parameters $\left(R_{1}, C_{1}, L_{3}\right.$ and $\left.R_{01}\right)$. Two cases have been considered: a first one where the value of the aforementioned parameters have an error about $10 \%$ on the nominal one, and a second case where this error is increased up to $20 \%$. Fig. 9 shows the different behaviours the voltage $V_{C_{2}}$ has according to the considered parameters' errors with respect to the nominal case. As it is also possible to state from Fig. 10, where the current $I_{L_{3}}$ is depicted with respect to the parameters' errors, to higher currents correspond higher errors. As for Fig. 6, the ripples in Fig. 9 caused by the converter switching are considered moderate.

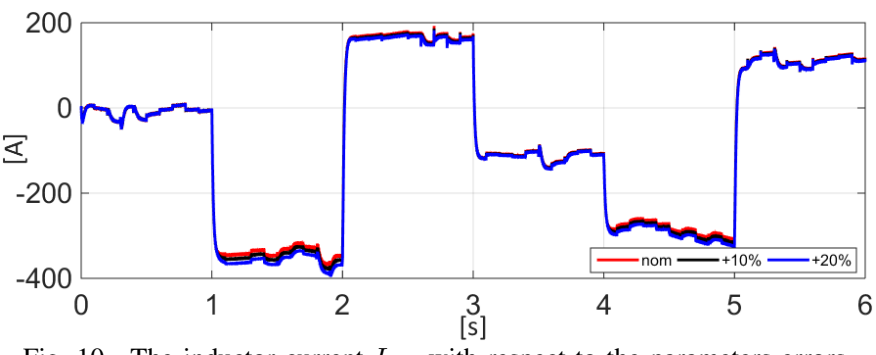

Fig. 10. The inductor current $I_{L_{3}}$ with respect to the parameters errors.

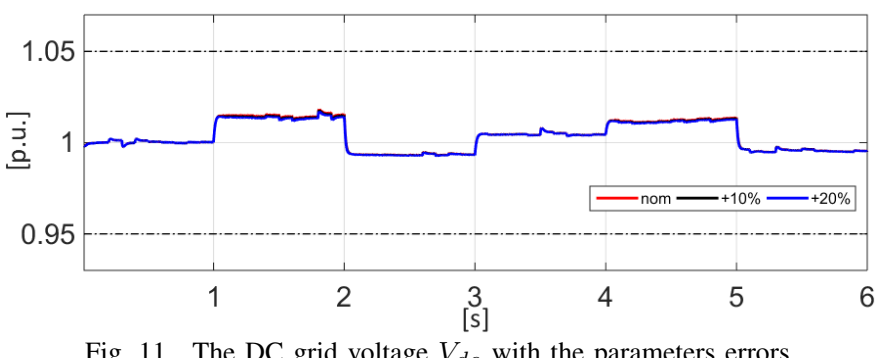

Fig. 11. The DC grid voltage $V_{d c}$ with the parameters errors.
Fig. 11 depicts the DC bus voltage and how the applied control is robust enough to regulate it for both the considered cases. Indeed, the parameters' errors do not affect the voltage to cause relevant changes in its behaviour.

\section{CONCLUSions}

In this paper a dynamic feedback linearization control strategy to regulate the voltage on the DC bus for a DC Microgrid is introduced. The proposed control action avoids problems related to non-minimum phase properties for power converters. Based on Thévenin equivalent circuit and singular perturbation analysis, hypothesis on the structure of the DC Microgrid are developed and tested, to the purpose to reduce control system complexity. Nevertheless, control system efficiency is ensured.

SimPowerSystem simulations illustrate how the simplified model can be successfully applied for control purposes. The robustness of the control strategy is tested in condition of parameters' values incertitude.

Both analytical and simulations results clearly show the capability of the proposed control scheme to stabilize the voltage of a DC Microgrid with good performance.

\section{REFERENCES}

[1] P. F. Ribeiro, B. K. Johnson, M. L. Crow, A. Arsoy, and Y. Liu, "Energy storage systems for advanced power applications," Proceedings of the IEEE, vol. 89, no. 12, pp. 1744-1756, 2001.

[2] D. E. Olivares, A. Mehrizi-Sani, A. H. Etemadi, C. A. Caizares, R. Iravani, M. Kazerani, A. H. Hajimiragha, O. Gomis-Bellmunt, M. Saeedifard, R. Palma-Behnke, G. A. Jimnez-Estvez, and N. D. Hatziargyriou, "Trends in microgrid control," IEEE Transactions on Smart Grid, vol. 5, no. 4, pp. 1905-1919, July 2014.

[3] X. She, A. Q. Huang, S. Lukic, and M. E. Baran, "On integration of solid-state transformer with zonal dc microgrid," IEEE Transactions on Smart Grid, vol. 3, no. 2, pp. 975-985, 2012.

[4] R. Teodorescu, M. Liserre, and P. Rodriguez, Grid converters for photovoltaic and wind power systems. John Wiley \& Sons, 2011, vol. 29.

[5] J. J. Justo, F. Mwasilu, J. Lee, and J.-W. Jung, "Ac-microgrids versus dc-microgrids with distributed energy resources: A review," Renewable and Sustainable Energy Reviews, vol. 24, pp. 387-405, 2013.

[6] K. Strunz, E. Abbasi, and D. N. Huu, "Dc microgrid for wind and solar power integration," IEEE Journal of Emerging and Selected Topics in Power Electronics, vol. 2, no. 1, pp. 115-126, March 2014.

[7] A. Iovine, S. B. Siad, G. Damm, E. D. Santis, and M. D. D. Benedetto, "Nonlinear control of a dc microgrid for the integration of photovoltaic panels," IEEE Transactions on Automation Science and Engineering, vol. 14, no. 2, pp. 524-535, April 2017.

[8] S. K. Kim and K. B. Lee, "Robust feedback-linearizing output voltage regulator for dc/dc boost converter," IEEE Transactions on Industrial Electronics, vol. 62, no. 11, pp. 7127-7135, Nov 2015.

[9] Y. Chen, G. Damm, A. Benchaib, and F. Lamnabhi-Lagarrigue, "Feedback linearization for the dc voltage control of a vsc-hvdc terminal," in 2014 European Control Conference (ECC), June 2014, pp. 1999-2004.

[10] A. Iovine, S. B. Siad, G. Damm, E. D. Santis, and M. D. D. Benedetto, "Nonlinear control of an ac-connected dc microgrid," in IECON 2016 - 42nd Annual Conference of the IEEE Industrial Electronics Society, Oct 2016, pp. 4193-4198.

[11] H. Sira-Ramírez and R. Silva-Ortigoza, Control design techniques in power electronics devices. Springer Science \& Business Media, 2006.

[12] Y. Zhang and Y. W. Li, "Energy management strategy for supercapacitor in droop-controlled dc microgrid using virtual impedance," IEEE Transactions on Power Electronics, vol. 32, no. 4, pp. 2704-2716, April 2017.

[13] H. Khalil, Nonlinear Control. Pearson Education, 2014. [Online]. Available: https://books.google.fr/books?id=OyGvAgAAQBAJ

[14] Y. Chen, G. Damm, A. Benchaib, and F. Lamnabhi-Lagarrigue, "Multitime-scale stability analysis and design conditions of a vsc terminal with dc voltage droop control for hvdc networks," in 53rd IEEE Conference on Decision and Control, Dec 2014, pp. 3266-3271. 